Revue européenne des sciences sociales

European Journal of Social Sciences

XXXVIII-119| 2000

La peur de l'impensable dans les sciences sociales

\title{
Le syndrome du précipice. La peur du vide symbolique
}

Henri Volken

\section{OpenEdition}

\section{Journals}

Édition électronique

URL : http://journals.openedition.org/ress/670

DOI : 10.4000/ress.670

ISSN : 1663-4446

Éditeur

Librairie Droz

Édition imprimée

Date de publication : 1 décembre 2000

Pagination : 23-33

ISBN : 2-600-00481-5

ISSN : 0048-8046

Référence électronique

Henri Volken, «Le syndrome du précipice. La peur du vide symbolique», Revue européenne des sciences sociales [En ligne], XXXVIII-119 | 2000, mis en ligne le 15 décembre 2009, consulté le 19 avril 2019. URL : http://journals.openedition.org/ress/670 ; DOI : 10.4000/ress.670 


\section{Henri VOLKEN}

\section{LE SYNDROME DU PRÉCIPICE. LA PEUR DU VIDE SYMBOLIQUE}

\section{Parcours:}

- Zéro et les nombres négatifs: le vide manipulé

- Point de fuite: l'infini à portée de main

- Les infiniment petits: double vertige

\section{- Les nombres imaginaires: le fantasme incarné}

Parmi les situations classiques de l'univers comique des bandes dessinées et des dessins animés, l'image à la fois terrifiante et irrésistible du précipice est l'une des plus typiques: un personnage s'enfuit à toutes jambes, se dirige vers un précipice qu'il ne voit pas et continue à courir bien qu'il se trouve déjà au-dessus du vide, sans contact avec la terre ferme. Il ne tombe pas.

Il ne tombera qu'au moment précis où il s'aperçoit qu'il est suspendu en l'air, au moment où il est conscient du précipice sous ses pieds! Alors la peur qui s'empare de lui, rend la chute encore plus spectaculaire, tout en amusant beaucoup le lecteur ou le spectateur qui avait anticipé la catastrophe retardée, grâce au recul que lui confère son statut d'observateur.

Nous allons tenter de mettre en lumière dans un autre domaine une forme analogue de ce mécanisme très simple, et de montrer en particulier que celui-ci jalonne également l'histoire des mathématiques. Ce schéma est visible en particulier aux moments clé, où la pratique des mathématiques essaye de domestiquer des notions aussi délicates et passionnément controversées que le zéro, l'infini et leurs avatars.

Dans le texte qui suit, nous allons essayer d'identifier ce mécanisme, que nous nommerons «syndrome du précipice» pour simplifier. On nous pardonnera ce petit clin d'œil à l'univers fictif, mais généreux et libre, des petits personnages dessinés. Après tout, le monde mathématique n'en est pas si éloigné, avec ses êtres imaginaires, légers, précis, parfois à la limite de l'existence.

Les situations que nous allons décrire pour illustrer notre propos ont toutes en commun une certaine forme de vertige, provoqué par la nouveauté et ses promesses d'une part, mais aussi, de l'autre, par la peur de l'inconnu et de la transgression. A travers ces quelques exemples historiques, nous tenterons de comprendre et de décrire le schéma de ce mécanisme qui n'épargne pas le monde mathématique, pourtant souvent jugé si éloigné des sentiments humains. 
Ce vertige - étymologiquement: mouvement tournant - nous le rencontrerons sous des aspects divers. Induit par l'apparition d'entités symboliques nouvelles qui semblent défier l'ordre établi, il peut être mouvement de retour en arrière, ou encore retournement de sens, ou simple chute imaginée. Mais toujours lui est associée une forme de peur. Est-ce la peur d'une sorte de vide conceptuel, ou estce un inconfort dû à une lacune sémantique, voire plus simplement la crainte d'engendrer la contradiction? Nous allons tenter de découvrir sa vraie nature à travers les exemples historiques suivants.

\section{I. - L'INTRODUCTION DU ZÉRO: LA MANIPULATION DU VIDE}

Comment imaginer aujourd'hui que l'utilisation du nombre zéro puisse poser un problème, ou pire, provoquer une peur et un rejet comparable à ceux qui ont accompagné les débuts de l'arithmétique dans l'Europe de la Renaissance? L'usage courant actuel confère au symbole «zéro» le même statut qu'aux autres nombres, à savoir qu'il représente une quantité, même si en l'occurrence celle-ci est nulle. Qu'une soustraction donne le résultat «zéro» ne dérange personne. Et même si on n'additionne pas volontiers «zéro» à un autre nombre, parce que cela nous paraît inutile, on admet que c'est possible et que le résultat en est cet autre nombre. Il est même communément accepté que les ordinateurs ne manipulent que des symboles «zéro» et «un». Or historiquement, l'introduction du «zéro», dans un climat de rejet, de crainte et d'incrédulité se trouve au début du développement de l'algèbre et de l'arithmétique, qui constituent le noyau des mathématiques que nous connaissons aujourd'hui. Avant d'analyser de plus près le problème du «zéro» et de voir comment son statut épistémique différent de celui des autres nombres en faisait un corps étranger, il est utile de se pencher brièvement sur la notion même de nombre et plus spécifiquement sur leur représentation.

Il y a de nombreuses spéculations sur l'origine des nombres, mais il semble que la notion de comptage soit principalement impliquée dans leur genèse. Pour rendre compte de cette activité, il suffit d'avoir à disposition un symbole (objet, lettre ou son) et d'itérer son emploi chaque fois qu'on passe au «successeur»: I, II, III, IIII, IIIII etc. Chaque étape de cette énumération devrait être désignée par un nom, pour qu'elle puisse servir à des fins de comparaison, ce qui est son but. Cela implique un nombre extrêmement grand de noms différents ou alors un système plus raffiné qui permette de créer des symboles nouveaux à chaque fois que c'est nécessaire. Ces systèmes raffinés de numération existent dans des civilisations très anciennes de l'Inde, de la Chine, du Moyen-Orient et de la Grèce notamment. Les plus anciens ont été inventés, il y a près de 5000 ans par les Sumériens, dont le célèbre système sexagésimal qui a laissé des traces jusque dans notre vie quotidienne, puisque notre minute possède 60 secondes et que notre heure compte 60 minutes. Ces systèmes de numération sont essentiellement de trois types: additif, hybride ou positionnel. Les deux premiers sont basés sur le principe d'addition, avec une valeur attachée à chaque chiffre, indépendamment de sa position dans la représentation. Les nombres romains en sont une illustration. Par exemple MCCXXXIIII est la représentation de $1000+100+100+10+10+10+1+1+1+1$ 
c'est-à-dire $1234^{1}$. Les systèmes additifs ou hybrides permettent de représenter un nombre de manière lisible, mais n'autorisent guère les opérations d'une certaine complexité: la multiplication de nombres écrits dans le système romain devient un casse-tête à partir déjà de nombres relativement petits: quelle procédure raisonnable faut-il appliquer par exemple pour passer des deux nombres XII et V à leur produit LX? Il n'y a de véritable calcul possible que dans le troisième type de représentation: le système positionnel que nous connaissons aujourd'hui. Ce système est basé sur l'idée que la valeur des chiffres est déterminée par leur position dans l'écriture des nombres. La représentation positionnelle des nombres, apparue sous une forme archaïque dans les systèmes babylonien, chinois mais aussi maya, nécessite l'usage d'un zéro, c'est-à-dire d'un symbole qui puisse marquer une place vacante dans l'ordre des chiffres. C'est le système indien, le plus raffiné et le plus performant, qui a non seulement intégré l'idée de position et le symbole «zéro» dans sa représentation des nombres, mais a également tenté de donner un sens à ce dernier symbole et de l'intégrer dans la catégorie des nombres véritables. Le terme «shûnya» était le nom ordinaire du zéro en sanskrit et signifiait «le vide», «l'absence», «le néant»ou «le rien». D’autres symboles numériques pour «zéro» pouvaient signifier «le point», «le trou», mais aussi «la totalité» et même «l'infini». Les mathématiciens indiens ont compris très tôt le lien entre le zéro et l'infini, l'un étant l'inverse de l'autre dans certains de leurs raisonnements impliquant la division. Le terme «shûnya» est devenu, en transitant par le monde arabe, «al sifr» puis finalement «chiffre» en français. «Chiffre» est devenu emblématique pour tous les symboles numériques indiens, ou «arabes» comme on les nomme parfois.

Lorsque la numération indienne fut progressivement adoptée en Europe, entre le XIIIe et le $\mathrm{XV}^{\mathrm{e}}$ siècle, elle fut mal accueillie par le clergé notamment ${ }^{2}$, qui craignait d'une part que la facilité de calcul que cette écriture offrait, lui enlèverait une partie de ses prérogatives en démocratisant trop un savoir qu'il détenait en partie. Mais d'autre part, c'est bien le zéro et son usage dans le calcul qui souleva les plus grandes craintes et les plus fortes réactions de rejet.

Les possibilités de calcul étant très limitées dans les systèmes de numération additifs, comme les chiffres romains, on s'aidait d'un abaque, sorte de planche avec des jetons que l'on manipulait selon des règles complexes. Certaines lignes des abaques pouvaient ne contenir aucun jeton et n'étaient donc pas représentées dans l'écriture puisque le «zéro» n'existait pas. Mais le concept «vide» ne posait aucun problème aux utilisateurs des abaques. Ceux-ci étaient appelés «abacistes», par opposition aux «algoristes ${ }^{3} »$ qui utilisaient l'écriture positionnelle avec le

Dans des versions plus tardives, une certaine influence de la position apparaît: au lieu de IIII on écrira IV avec la convention qu'une valeur inférieure qui précède une valeur supérieure doit être soustraite de celle-ci.

2 Pourtant c'est Gerbert d'Aurillac, le futur pape Sylvestre II, qui a tenté d'introduire les chiffres arabes (sans le zéro) en Europe au début du millénaire. Mais il fut traité plus ou moins ouvertement de «sorcier» et d' "alchimiste», et en 1648 son tombeau fut même ouvert sur ordre des autorités pontificales pour vérifier s'il contenait des preuves de sorcellerie.

3 En référence au mathématicien arabe $\mathrm{Al}$ Khuwarizmi, dont le livre d'arithmétique résumait le système indien et permit sa diffusion dans le monde arabe. Le terme «algorithme» que nous connaissons aujourd'hui dérive de son nom. 
«zéro». L'écriture indienne permettait aux algoristes de calculer directement à partir des chiffres, selon des règles simples et efficaces. Cependant l'usage du symbole «zéro» devint rapidement la source d'une certaine peur dont l'origine est à chercher dans l'ambiguïté sémiotique de ce nouveau signe. D'une part, il signalait dans l'écriture positionnelle l'absence d'autres signes. Il était donc un metasigne et semblait d'une autre nature que les chiffres de 1 à 9 . Or d'autre part il se comportait dans les calculs comme ces derniers, puisqu'il pouvait entrer dans les opérations arithmétiques courantes. On pouvait même le considérer comme un opérateur, puisque le fait de l'écrire à la suite d'un nombre multipliait celui-ci par dix. Mais les chiffres usuels représentaient des «quantités» et possédaient donc une certaine réalité que le «zéro»n'avait pas ${ }^{4}$.

La peur engendrée par les algoristes est de la famille du syndrome du précipice: en partant d'un problème concret on voyait ceux-ci manipuler des symboles, dont certains ne représentaient «rien», pour aboutir comme par enchantement, avec facilité, à des résultats qui étaient corrects! La «sorcellerie» consistant à marcher au-dessus du vide pendant quelques instants pour retrouver la terre ferme avec aisance. Ce type de situation va se retrouver à de nombreuses reprises dans l'histoire des mathématiques.

Pourtant si le symbole «zéro», à la fois ambigu, sémantiquement incertain mais aussi diaboliquement efficace, est à l'origine d'une peur répandue, son usage est aussi le point de départ d'un développement prodigieux de l'arithmétique et de l'algèbre. C'est précisément cette peur qui a provoqué l'apparition d'une nouvelle conception des nombres, plus abstraite et plus unifiée. Les nombres acquéraient une autonomie par rapport aux objets préexistants qu'ils étaient supposés représenter jusque-là. Le mathématicien hollandais Simon Stevin parle même du zéro comme de l' «arché» des nombres, leur véritable origine, ce qui est une idée qui anticipe de nombreux progrès plus récents. Par l'attention qu'elle a mobilisée sur un problème précis, la peur suscitée par l'introduction du «zéro» est donc le véritable catalyseur de ce processus de réajustement sémantique.

\section{II. - L'INVENTION DE LA PERSPECTIVE: L'INFINI À PORTÉE DE MAIN}

La tradition mathématique indienne utilisait, nous l'avons évoqué plus haut, de nombreux symboles pour désigner le «zéro», dont certains, comme «ananta ${ }^{5}$ », représentaient littéralement l'«infini» entraînant une certaine confusion terminologique. Un autre nom indien pour l'infini, «khahara», signifie mot à mot: «quantité dont le diviseur est zéro». La culture mathématique de l'Inde avait donc établi le lien entre zéro et infini, en découvrant que l'un était l'inverse de l'autre pour l'opération de la division. Ce lien opératoire mais aussi d'autres liens plus mystiques reliaient déjà ces deux concepts apparemment antagonistes. Tous deux exerceront un effet de fascination et de peur à plusieurs reprises lors de l'appari-

\footnotetext{
Encore que le «1» ne semblait pas non plus représenter vraiment une «quantité».

5 Le serpent couché qui se mord la queue, et qui représente ananta, est devenu le symbole contemporain pour l'infini.
} 
tion d'objets mathématiques nouveaux. Si depuis ses premières utilisations, on avait attaché le sens de «vide», de «néant» et de «rien» au symbole «zéro», la signification des symboles pour l' «infini» n'était pas aussi univoque. Infini était avant tout un terme que les théologiens utilisaient comme attribut divin. En Europe, avant de faire son apparition dans l'arsenal des mathématiciens, l'«infini» fit irruption, en tant que signe, dans le monde de la peinture de manière surprenante et troublante.

Dans une expérience célèbre, le peintre et architecte florentin Brunelleschi établit les bases de l'art de la perspective qui devait bouleverser le développement de la peinture. Les parallèles orthogonales au plan du tableau par exemple n'étaient plus représentées dans le tableau par des droites parallèles, mais devenaient sécantes et se coupaient en un point, appelé point de fuite. Ce point mystérieux est à l'origine d'une vision de l'espace radicalement transformée: la profondeur ajoutait une nouvelle dimension au tableau lui conférant un réalisme saisissant. Tous les points du tableau pouvaient être perçus avec leur distance. Mais le point de fuite, étant théoriquement à l'infini, ne pouvait correspondre à rien! Tous les points du tableau avaient donc un «correspondant» réel, sauf le point de fuite qui n'avait pas de «sens» puisqu'il ne représentait rien. S'il était un point du tableau, il signifiait principalement l'absence d'un point réel, un peu comme le «zéro» signifiait l'absence d'un chiffre. On trouve ici la même ambiguïté sémiotique qui est à l'origine d'une peur identique: comment admettre que l'on manipule un objet symbolique, ici un point sur le tableau, qui représente une réalité impossible? Le point de fuite qui permettait de représenter la profondeur d'une scène picturale, permettait aussi en effet de mettre le doigt sur l'infini et rendait visible un point inexistant. Cette possibilité de ramener un point à l'infini sur le plan du tableau provoquait le même malaise que celle qui consistait à manipuler un symbole qui correspondait au «vide».

Les peintres de la Renaissance ont soigneusement évité de montrer le point de fuite. Il est le plus souvent caché par un personnage, ou par un accessoire du décor. Parfois il est rejeté dans l'incertain d'un miroir ou dans le lointain au travers d'une fenêtre ouverte. Jamais il n'apparaît explicitement fixé en tant que point sur un tableau.

La peur provoquée par le point de fuite ne s'explique pas uniquement par le fait que ce point possède la troublante identité double de signe et de meta-signe, mais également parce qu'il est en quelque sorte ${ }^{6}$ le symétrique du point de vue, ce point théorique où se situe le spectateur mais aussi l'artiste. Les deux points sont confondus sur le tableau, alors que dans l'espace réel à trois dimensions, l'un est situé à une distance finie, devant le tableau - c'est la position de l'observateur - et l'autre derrière le tableau à une distance infinie. Cette confusion peut être à l'origine d'un vertige durant lequel le spectateur est attiré par l'infini tout en occupant la position du point de vue. Le premier indique l'œil de l'artiste et le second à la fois signale un vide et renvoie à l'infini, rappelant par-là le rapprochement du zéro et de l'infini dans la terminologie mathématique indienne.

La crainte de la part des peintres, d'exercer leur pouvoir tout neuf de ramener l'infini dans les limites du tableau, qui se traduit par divers stratagèmes de dissi-

6 Dans un sens métaphorique uniquement. 
mulation, n'a pas éveillé immédiatement la curiosité des mathématiciens. Ce n'est que deux siècles plus tard que l'idée de projection qui est à la base de la technique de la perspective, a permis plus tard à Desargues et Pascal au XVII ${ }^{\mathrm{e}}$ siècle de développer la géométrie projective, dans laquelle tous les points sont de même nature, y compris les points à l'infini, qu'une projection peut rendre accessibles. La situation est analogue à celle que l'introduction du «zéro» a provoquée dans l'univers du nombre: une redéfinition de la sémantique des objets mathématiques en présence qui les rend tous semblables.

\section{III. - LES INFINIMENT PETITS: UN DOUBLE VERTIGE}

Toutes les peurs décrites jusqu'ici concernent l'introduction d'objets symboliques par lesquels on semble manipuler - de manière trop simple - des concepts dangereux comme le «rien» et l'«infini». L'exemple suivant combine ces deux notions et illustre une tension particulièrement forte. Il s'agit de l'introduction des infiniment petits de manière indépendante par Newton et Leibniz au XVII siècle. La nécessité de trouver des équations définissant les tangentes à une courbe est la motivation pour la création de ces êtres mathématiques étranges, les infinitésimaux, ou les infiniment petits. Introduits tout d'abord par Newton sous le terme de «fluxions», ensuite par Leibniz sous le nom de «différentielles», les infiniment petits se caractérisent par le fait qu'ils créent une sorte de halo autour du nombre zéro, formé de grandeurs qui ont partiellement les propriétés des nombres en ce sens qu'on peut les additionner, les soustraire, les multiplier et même les diviser. Mais ils sont d'un ordre de grandeur différent des nombres, et ne satisfont pas, par exemple, l'axiome d'Archimède. Celui-ci stipule, dans la formulation d'Euclide', que:

« Des grandeurs sont dites avoir un rapport l'une relativement à l'autre, quand elles sont capables, étant multipliées, de se surpasser l'une l'autre. »"

Or précisément les infiniment petits n'ont pas cette propriété: jamais un infinitésimal, multiplié par n'importe quel nombre, n'atteindra ou ne dépassera un nombre différent de zéro. Plus grave encore, le carré d'un infinitésimal est «négligeable» ou «pas différent de zéro» mais sans être nul!

Voici une illustration de la manière de raisonner décrit dans le calcul de Leibniz. L'une des motivations pour l'introduction de ces méthodes était la détermination des tangentes à une courbe, par exemple à la parabole $y=x^{2}$. En choisissant un point et un accroissement infinitésimal $d x$, la différentielle se calcule par la formule $d y=\left(x_{0}+d x\right)^{2}-x_{0}^{2}$. Cette expression devient $d y=x_{0}^{2}+2 x_{0} d x+d x^{2}-x_{0}^{2}=$ $2 x_{0} d x+d x^{2}$. Or dans la logique des infiniment petits, les carrés d'un tel «nombre» sont assimilés à zéro. On avait donc $d y=2 x_{0} d x$. Dans le monde infinitésimal, la courbe était linéarisée! La tangente «apparaissait» comme par miracle, rendue visible par une petite concession à la vraisemblance: il suffisait d'admettre qu'il existait des entités à la fois nulles et non-nulles.

Un tel comportement, qui associait «infini» et «zéro» à travers un halo infini de grandeurs infiniment proches de zéro, ne pouvait qu'inquiéter les âmes sen-

Euclide: Les Eléments, livre V, définition 4. 
sibles, qui admettaient pourtant que leur utilisation faisait merveille dans les problèmes concrets, grâce notamment au calcul des infinitésimaux développé par Leibniz, qui rendait mécaniques certaines opérations complexes. Puis, après une phase véritablement euphorique de leur utilisation, en particulier dans la physique de Newton, la conscience du vide sémantique se manifesta. L'un des critiques les plus virulents de ces nouveaux objets mathématiques était le philosophe et théologien G. Berkeley, farouche adversaire des infiniment petits qui échapperaient à la perception ${ }^{8}$. Son ironie allait jusqu'à traiter les infiniment petits de «souvenirs de grandeurs disparues »! Ici la peur, ou la dissonance, était engendrée par un double vertige, provoqué par une nouvelle forme de zéro, différente du nombre zéro, mais définie par une infinité d'êtres au comportement étrange et au statut ontologique ambigu. Le résultat de cette réaction était tout d'abord une période de refoulement. En effet, l'introduction de la notion de limite par les mathématiciens allemands du XIX ${ }^{\mathrm{e}}$ siècle rendait théoriquement superflue l'utilisation des infiniment petits. Dans la pratique on continuait cependant à les utiliser en profitant de la simplicité de leur formalisme et de l'élégance des règles du calcul qui les géraient. Le vertige avait disparu puisque la notion de limite permettait de donner une base conceptuelle plus solide au domaine de l'analyse mathématique. Cette période de refoulement des infiniment petits n'a pris fin que récemment - il y a un peu plus de trente ans - avec l'apparition de la théorie de l'analyse non-standard', qui justifie l'emploi des grandeurs infinitésimales et établit la légitimité des opérations introduites par Newton et par Leibniz au XVII ${ }^{\mathrm{e}}$ siècle. Cette théorie constitue la résolution de la tension provoquée par ces étranges objets que sont les infinitésimaux, puisque sa consistance logique est formellement établie. En effet, pour la plupart des mathématiciens, la consistance d'une théorie implique l'existence des objets qu'elle décrit. Il est à remarquer que la réhabilitation des infiniment petits n'a cependant pas eu d'influence sur l'enseignement des mathématiques, où l'on se prive toujours de la possibilité, indiquée par Robinson, de réduire, d'une certaine manière, l'analyse à l'algèbre. La peur n'a pas entièrement disparu dans l'esprit des enseignants qui préfèrent le lourd et parfois obscur formalisme des limites, à l'élégance du calcul Leibnizien enfin légitimé.

\section{IV. - LES NOMBRES IMAGINAIRES: LE FANTASME INCARNÉ}

Dans le courant du XVI ${ }^{\mathrm{e}}$ siècle, l'arithmétique fit un grand pas en avant grâce au monumental traité de Geronimo Cardano, Ars magna. Ce traité contenait toute la science arithmétique accumulée jusque-là, et de nombreuses découvertes récentes, en particulier les méthodes de résolution des équations cubiques et quadratiques qui constituaient l'un des progrès mathématiques majeurs depuis l'époque babylonienne. Les idées originales de ces solutions n'étaient d'ailleurs pas de Cardano lui-même, mais de Tartaglia et Ferrari. La date de parution de ce livre, 1545 , est souvent citée comme le début de la période moderne de l'histoire des

\footnotetext{
Notamment dans The Analyst, 1734

9 Dans un livre d'Abraham Robinson: Non-standard Analysis, Amsterdam 1966.
} 
mathématiques. L'exemple de la résolution des équations cubiques nous permet d'illustrer un nouvel aspect du syndrome du précipice. Par une habile substitution, Cardano parvint à réduire le cas général à la forme normale $x^{3}+p x=q$. Puis dans ce cas-là, on trouvait une solution par la nouvelle méthode en calculant:

$$
x=\sqrt[3]{\sqrt{(p / 3)^{3}+(q / 2)^{2}}+q / 2}-\sqrt[3]{\sqrt{(p / 3)^{3}+(q / 2)^{2}}-q / 2} .
$$

Mais cette procédure pouvait parfois mener à des impasses. Prenons l'exemple suivant. L'application directe de la méthode nous donne:

$$
x=\sqrt[3]{2+\sqrt{-121}}+\sqrt[3]{2-\sqrt{-121}}
$$

opération qui devait être considérée comme «impossible» d'après les canons de la science de l'époque, le carré d'un nombre étant forcément non-négatif. Il n'y avait donc pas de racines de nombres négatifs. Néanmoins la réponse par ailleurs évidente, $x=4$, était connue. Mais comment identifier l'expression obtenue avec le nombre 4? On pouvait bien sûr réduire l'expression à:

$$
x=\sqrt[3]{2+11 \sqrt{-1}}+\sqrt[3]{2-11 \sqrt{-1}}
$$

par les règles de calcul connues, mais la difficulté subsistait. La motivation pour tenter de donner un sens à ces objets nouveaux était claire: on voulait que la méthode de résolution donne une réponse dans tous les cas de figure. C'est ici que le mathématicien italien R. Bombelli eût une intuition particulièrement hardie, une «pensée sauvage» selon ses propres termes, qui allait révolutionner le monde mathématique:

Supposons que $\sqrt{-} 1$ existe mais soit d'une autre nature que les nombres habituels!

On peut alors chercher une solution qui participe de cette double nature, c'està-dire qui puisse s'écrire comme somme de deux nombres, l'un étant un nombre habituel et l'autre un multiple de ce fantasme désigné par $\sqrt{-1}$. Il est ensuite aisé de voir qu'un tel «nombre» qui soit la racine cubique de $2+11 \sqrt{-}-1$, ne peut avoir que la forme $2+\sqrt{-}$. La solution devient ainsi $x=2+\sqrt{-1}+2-\sqrt{-1}=4$, expression dans laquelle la partie litigieuse disparait! C'est-à-dire qu'on retrouve de cette manière la solution espérée, celle qui est représentée par un nombre «habituel». Que s'est-il passé? La fiction d'un nombre $\sqrt{-} 1^{10}$, qui ne peut pas exister d'après les principes admis, a permis de trouver une solution parfaitement réelle. Le prix à payer est un passage mystérieux par un monde imaginaire, totalement inconnu! Il faut admettre également que toutes les manipulations s'y font d'après des règles connues, mais impliquent des êtres dont on sait qu'ils n'existent pas. Mais à la sortie, la réponse obtenue semble récompenser l'audace!

Telle était la démarche aventureuse des algébristes de la seconde moitié du $\mathrm{XVI}^{\mathrm{e}}$ siècle, trop heureux d'avoir trouvé un outil efficace pour résoudre systématiquement des équations réputées difficiles. L'introduction de ces grandeurs au statut ontologique des plus incertains, ouvrait en outre un champ immense de possibilités pour le développement de méthodes algébriques nouvelles.

10 Ce nombre fut appelé «imaginaire» par la suite. On le remplace la plupart du temps par le symbole «i». 
Mais la dissonance était présente. Malgré le succès obtenu par leur utilisation, les nouveaux nombres «imaginaires» créaient une certaine peur, car ils étaient perçus comme dangereux, probablement à cause de leur création à partir d'une insolente contradiction. Et même Descartes et Leibniz se méfiaient beaucoup de leur statut, bien que Leibniz ait apporté quelques contributions à leur utilisation, montrant par exemple que

$$
\sqrt{6}=\sqrt{1}+\sqrt{-3}+\sqrt{1}-\sqrt{-3}
$$

une décomposition d'un nombre réel qui étonna ses contemporains. Leibniz, qui était également théologien, comparait les nombres imaginaires à des grandeurs amphibies entre être et non-être: «...idealis mundi monstro, pene inter Ens et nonEns Amphibio, quod radicem imaginariam appellamus $\rangle^{11}$. Un des principaux facteurs de la tension créée par l'apparition de ces nouvelles entités, réside dans l'absence de représentation qu'on pouvait se faire des nombres imaginaires. Les nombres habituels, réels, étaient représentés comme points sur une droite ordonnée, mais où fallait-il situer les nombres imaginaires?

La phase de consonance ne commencera qu'un siècle plus tard. La notion de nombre complexe, intégrant les réels et les imaginaires, accordait une existence légitime, bien que toujours contre intuitive, à des êtres mathématiques issus d'un paradoxe. Gauss ${ }^{12}$ en fit un usage décisif en les intégrant de manière essentielle dans son théorème fondamental de l'algèbre qui affirme que chaque fonction polynomiale possède une racine complexe. Voici ce qu'il dit dans ses «Recherches arithmétiques» publiées en 1801:

\begin{abstract}
... mais les quantités imaginaires, autrefois et parfois encore maintenant appelées faussement impossibles, quand on les opposait aux quantités réelles, sont tolérées plutôt que complètement admises. Elles paraissent former un jeu vide de symboles, auxquels un substrat de pensée est refusé sans hésitation, même par ceux qui ne déprécient pas l'importante contribution de ces symboles au trésor des relations entre quantités réelles.
\end{abstract}

En même temps le problème de la représentation des nombres complexes était résolu en leur faisant correspondre les points d'un plan, appelé aujourd'hui le plan de Gauss. On pouvait ainsi «voir» ${ }^{13}$ tous les nombres, réels ou imaginaires, et cette représentation supprimait en partie le malaise créé par une naissance contradictoire: le fantasme s'était incarné. Les succès obtenus plus tard par des applications en électricité et en mécanique achevèrent d'imposer les nombres imaginaires au rang d'outils indispensables. Mais pour une partie des utilisateurs, des relents de magie subsistent aujourd'hui encore sous leur aspect de parfaite mécanique.

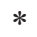

Le syndrome du précipice constitue l'un des schémas de la manifestation de la peur dans le monde des symboles et des représentations abstraites. De quelle

\footnotetext{
11 Ecrits mathématiques, V, 357. (1702)

12 C.F. Gauss, mathématicien allemand, (1777-1855)

13 Il s'agit d'une extension de la vision des nombres réels comme points d'une droite.
} 
nature peut être cette peur? Les illustrations qui précédent et qui sont tirées de l'histoire des mathématiques suggèrent au moins trois réponses possibles.

La manipulation d'un objet symbolique sans référence peut créer un vertige devant la possible contamination par le vide. Car si l'un des symboles n'a pas de référence, on peut craindre qu'une combinaison dans laquelle il entre perde son interprétation sémantique. Si dans un discours sur une figure géométrique, disons le cercle, on se focalise sur le «cercle carré» ${ }^{14}$, la suite des considérations risque de n'avoir plus de lien évident avec la réalité géométrique.

En plus de la perte de sens, on peut craindre aussi une disparition de la cohérence par l'introduction d'objets symboliques non interprétés. En effet, des objets portant en eux une certaine contradiction, comme ceux que nous avons rencontrés, pourraient apparemment affecter la cohérence logique du discours dans lequel ils interviennent. Si l'expression nous paraît contradictoire en elle-même, comment ne pas craindre que les opérations arithmétiques dans lesquelles elle intervient, ne mènent à des aberrations?

Une troisième forme de la peur est beaucoup plus irrationnelle. Elle concerne l'aspect d'opération magique que constitue toute manipulation de symboles vides de sens et incohérents, mais qui néanmoins parvient, parfois à travers la solution de problèmes particulièrement difficiles, à ouvrir des champs entièrement nouveaux. La perspective d'un changement de paradigme imposé par des concepts aussi incompréhensibles, ne peut qu'attiser une inquiétude diffuse à propos d'opérations abstraites pouvant intégrer si facilement et avec succès des fictions invraisemblables.

Le syndrome du précipice peut donc finalement être caractérisé par le schéma suivant qui en décrit les étapes essentielles ${ }^{15}$ :

Une première phase dans laquelle apparaît la motivation pour l'introduction d'un symbole nouveau.

Elle est suivie d'une phase d'euphorie où les premiers succès opératoires semblent justifier pleinement l'utilisation de ce nouvel objet.

Une phase ensuite de dissonance, durant laquelle apparaît son ambiguïté ontologique, provoquant une peur du vide sémantique, et en même temps un vertige devant les possibilités nouvelles, offertes au prix d'une conversion qui peut sembler douloureuse.

Dernière phase enfin, la résolution, qui montre que cette conversion, bien que représentant un bouleversement conceptuel, peut être pleinement justifiée rationnellement par une théorie qui englobe plus largement le domaine et apporte plus d'explications.

Paradoxalement, une certaine appréhension, pouvant aller jusqu'à l'angoisse parfois, induite par des objets mathématiques insolites, peut contribuer grandement au développement d'une théorie meilleure par l'attention qu'elle attire sur un problème précis. Il faut pour cela que le vertige, provoqué par une dissonance

4 Cf. la théorie des objets impossibles de Meinong.

15 Il faut rapprocher ce schéma des diverses tentatives épistémologiques de traiter les notions de «changement» et de «révolution» scientifiques. Nous renvoyons le lecteur aux travaux de Kuhn, Lakatos bien sûr, mais aussi à ceux, moins connus, de J.D. Sneed, W. Stegmüller et C. Dilworth. (cf. bibliographie). 
sémantique, puisse être surmonté. La peur peut ainsi favoriser notre compréhension du monde.

La chute ne correspond pas, dans le syndrome du précipice, à celle des dessins animés. Le scénario s'y poursuit de la manière suivante: la peur provoque une reconsidération du vide, une nouvelle interprétation du danger encouru, avec le résultat que la course au-dessus du précipice semble continuer sur un pont suspendu, dont l'existence serait révélée à travers le brouillard par une surprenante extension sémantique des concepts incriminés. Les exemples qui nous ont permis d'éclairer ce phénomène sont certes tirés de l'histoire des mathématiques, mais concernent par leur généralité et leur simplicité toute l'histoire de la pensée.

Le mécanisme ainsi décrit permet de réexaminer les quelques exemples de peur symbolique présentés, et bien d'autres, en rendant visible la parenté qui les relie. Il montre aussi que la peur et la dissonance qui lui sont associées préparent la résolution, cette phase capitale dans la création d'une nouvelle théorie plus générale.

Ce schéma n'est pas propre aux mathématiques. Il apparaît également dans les grands systèmes symboliques que sont les arts. Il suffit de penser à la musique ou à la peinture. L'apparition de la musique dodécaphonique et l'avènement de la peinture abstraite au début de ce siècle pourraient en constituer de bons exemples.

La peur du vide symbolique, celle qui nous fait craindre l'incohérence, se révèle, à travers ce schéma, être un important stimulant pour la recherche d'explications nouvelles, qui rendent notre monde de plus en plus lisible. Cette peur nous touche cependant de manière beaucoup plus essentielle, bien au-delà des préoccupations purement intellectuelles que semblent constituer nos jeux et manipulations de signes. Et c'est probablement dans la peur des mathématiques d'une partie des enfants, que se révèle le plus clairement la véritable dimension d'une angoisse sourde, provoquée par nos insolentes tentatives d'apprentis sorciers dans le monde des symboles.

Institut de mathématiques appliquées

Université de Lausanne

\section{BIBLIOGRAPHIE SOMMAIRE}

Dilworth, C.: Scientific Progress, Dordrecht, 1986.

Ifrah, G.: Histoire universelle des chiffres, Paris, 1994.

Kuhn, T.S.: The Structure of Scientific Revolutions, Chicago, 1970.

Panofsky, E.: La perspective comme «forme symbolique», Paris 1975.

Sneed, J.D.: The Logical Structure of Mathematical Physics, Dordrecht, 1979.

Stegmüller, W.: The Structure and Dynamics of Theories, New York, 1976. 